\title{
Nonsurgical management of traumatic cardiac pseudoaneurysms
}

\author{
Rashid M. Ahmad, MD, Ramanan Umakanthan, MD, Mark Lawson, MD, Marzia Leacche, MD, \\ Nataliya V. Solenkova, MD, and John G. Byrne, MD, Nashville, Tenn
}

Present literature has recommended surgical intervention to treat cardiac pseudoaneurysms. We describe the nonoperative management of a patient in whom imaging studies strongly suggested a traumatic right ventricular pseudoaneurysm. The findings resolved spontaneously on serial imaging studies.

\section{CLINICAL SUMMARY}

A 50-year-old man presented to the emergency department (ED) after being involved in a motor vehicle collision. He had undergone coronary artery bypass grafting 10 years previously. In the ED, he was intubated and bilateral chest tubes were placed for bilateral pneumothoraces. Injuries included an extensive scalp laceration that was repaired in the ED, bilateral pulmonary contusions, bilateral rib fractures, right femur fracture, and right tibia/fibula fracture. Computed tomography scan of the chest, performed on arrival, showed a $5 \times 8$-cm anteroposterior $\times$ left-right $(\mathrm{AP} \times \mathrm{LR})$ pseudoaneurysm of his right ventricle (RV). At the time of admission, the patient's systolic blood pressure was $70 \mathrm{~mm} \mathrm{Hg}$ and pulse rate was 108 beats/min. Physical examination revealed a fractured sternum in multiple locations, palpable as numerous floating islands. Vasopressors were necessary to sustain an adequate perfusion pressure. Twelve units of blood were transfused within the first 7 hours of admission. Echocardiography performed on admission demonstrated an akinetic and dilated RV infundibulum. Continuous color Doppler flow was observed within the RV outflow tract, suggesting blood flow into an RV pseudoaneurysm. No tricuspid regurgitant jet was seen to suggest severe pulmonary hypertension, which would increase the concern for pseudoaneurysm rupture. The echocardiogram showed no evidence of pericardial effusion or tamponade. Given the patient's degree of hemodynamic instability, which was attributed to blood loss from his multiple long bone fractures, we concluded that any immediate cardiac intervention would entail prohibitively high operative risk. We were also concerned that reoperative sternotomy and subsequent closure would be

\footnotetext{
From the Vanderbilt Heart and Vascular Institute, Nashville, Tenn.

Disclosures: None.

Received for publication Sept 5, 2008; accepted for publication Dec 24, 2008; available ahead of print June 1, 2009.

Address for reprints: John G. Byrne, MD, Vanderbilt Heart and Vascular Institute, 1215 21st Avenue South, Nashville, TN 37232-8802.

J Thorac Cardiovasc Surg 2010;139:e83-5

$0022-5223 / \$ 36.00$

Copyright (c) 2010 by The American Association for Thoracic Surgery

doi:10.1016/j.jtcvs.2008.12.055
}

challenging because of the complex nature of his comminuted sternal fracture. Therefore, the patient was closely monitored and resuscitated. An echocardiogram to assess for evolution of the cardiac injury was repeated the next day and displayed no pericardial effusion, tamponade, or increase in pseudoaneurysm size. The patient was weaned from vasopressors within 5 days. Orthopedic surgery performed open reduction and internal fixation of his orthopedic injuries 1 week after admission. Cardiac magnetic resonance imaging (MRI) performed 2 weeks after his injury (Figure 1) demonstrated a $5.9 \times 8.2 \mathrm{~cm}(\mathrm{AP} \times \mathrm{LR}) \times 6.4 \mathrm{~cm}$ craniocaudal (CC) pseudoaneurysm of the RV infundibulum that did not involve the pulmonic annulus or main pulmonary artery. The patient continued to improve clinically and was discharged from the hospital 3 weeks after admission with plans for an outpatient cardiac MRI. Follow-up MRI performed 3 months after presentation showed that the pseudoaneurysm had decreased in size to $2.3 \times 2.9 \times 2.3 \mathrm{~cm}(\mathrm{AP} \times \mathrm{LR} \times$ CC) and by 8 months after presentation had nearly resolved with only a small $1.5-\mathrm{cm}$ dyskinetic portion of the anterior infundibulum (Figure 2).

\section{DISCUSSION}

Current literature promotes surgical intervention for traumatic cardiac pseudoaneurysms because of the concern for rupture. ${ }^{1-3}$ However, it does not distinguish between treatment options for traumatic right versus left ventricular pseudoaneurysms. In contrast with left ventricular pseudoaneurysms, right ventricular pseudoaneurysms may be amenable to nonoperative management because of the lower transmural pressure of the right-sided circulation. Laplace's Law states that ventricular wall stress $(\sigma)$ is directly related to transmural pressure $(P)$ and ventricular chamber radius $(R)$, and inversely related to ventricular wall thickness $(h)$ :

$$
\sigma=\frac{P R}{2 h} \quad \text { LaPlace's Law }
$$

Thus, right ventricular wall stress is low given the low pressure characteristic of the right side of the heart. The risk of pseudoaneurysm rupture, therefore, will be less in comparison with traumatic left ventricular pseudoaneurysms. An important exception would be the presence of severe pulmonary hypertension, which would increase the risk of rupture because of higher right ventricular peak systolic pressures. Our patient had no evidence of severe pulmonary hypertension. Moreover, he had a history of cardiac surgery. Retrosternal fibrosis and adhesions from the previous 

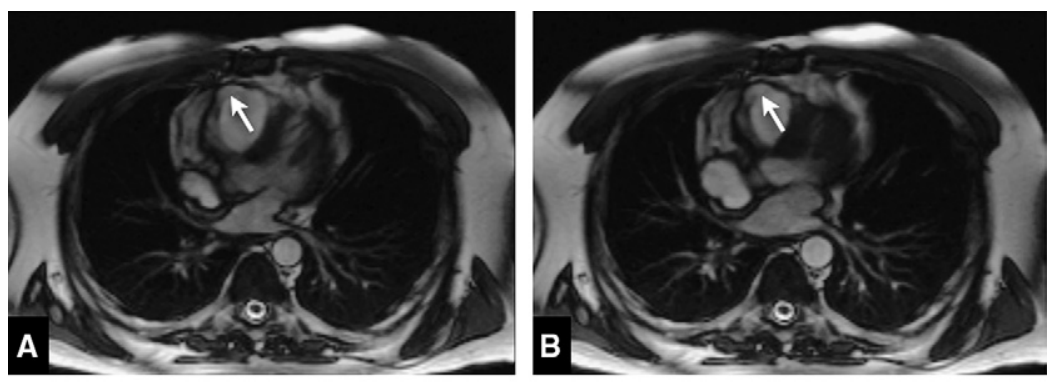

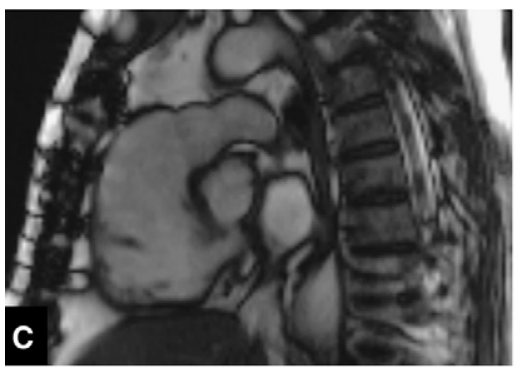

Diastole

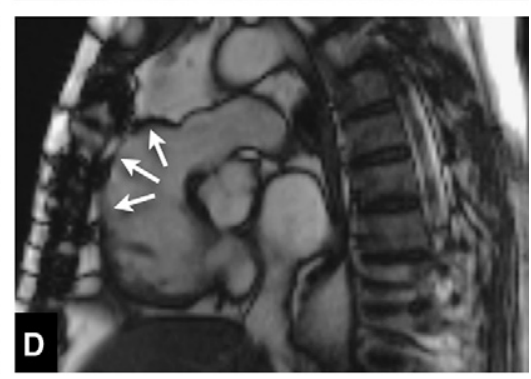

Systole

FIGURE 1. Axial (A, B) and oblique sagittal (C, D) views of the heart on cardiac MRI, showing the location and extent of the right ventricular pseudoaneurysm (arrows) during diastole (A, C) and systole (B, D). Note the marked thinning and akinesis of the anterior free wall of the infundibulum.
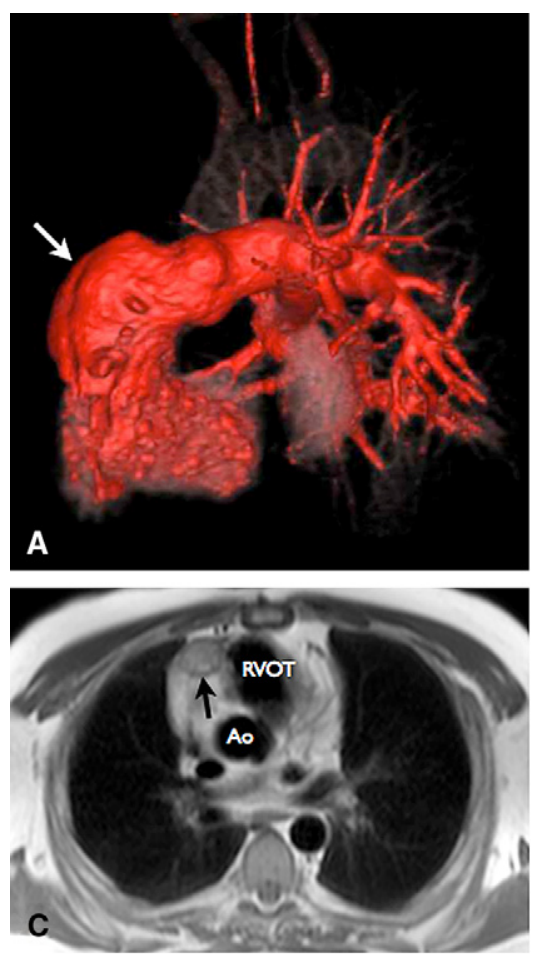

During Hospital Admission
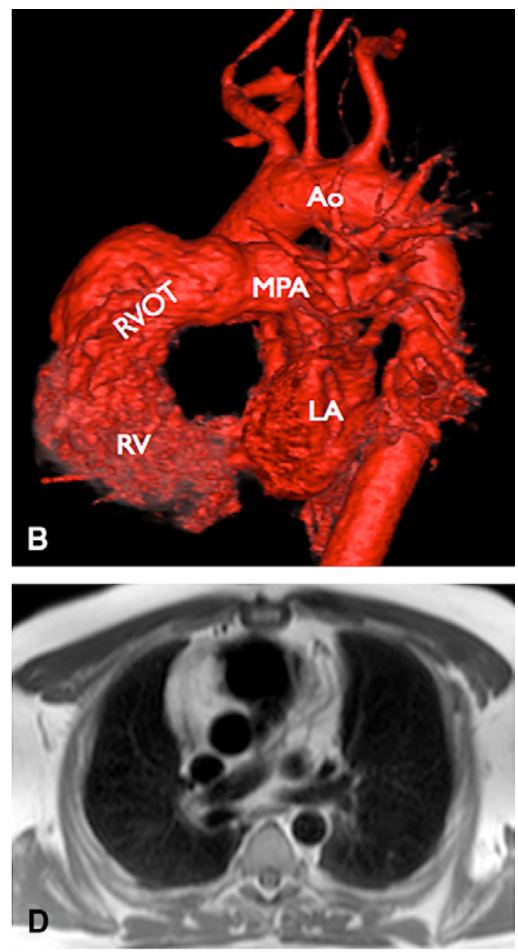

Eight Months After Injury

FIGURE 2. Sagittal 3-dimensional reconstructed views of the heart and great vessels on cardiac magnetic resonance angiography performed during hospital admission (A) and 8 months after injury (B). The pseudoaneurysm is shown by the white arrow. Axial dark blood cardiac MRI views of the heart performed during hospital admission (C) and 8 months after the injury (D). Slow flow is demonstrated in the pseudoaneurysm (black arrow) on the initial study. Note the decrease in pseudoaneurysm size on the 8-month follow-up study. Ao, Aorta; $R V$, right ventricle; $R V O T$, right ventricular outflow tract; $L A$, left atrium; $M P A$, main pulmonary artery. 
surgery provided tissue stabilization adjacent to the infundibulum, prevented pseudoaneurysm expansion, and minimized risk of rupture. We hypothesize that low right ventricular pressures and containment of the pseudoaneurysm by scar tissue from prior cardiac surgery allowed time for healing and obviated the need for surgery.

\section{CONCLUSIONS}

In selected patients with imaging studies strongly suggestive of traumatic RV pseudoaneurysms and a history of sternotomy, nonoperative management with close monitoring may be considered in the absence of tamponade, increase in pericardial effusion, severe pulmonary hypertension, deterioration in hemodynamic status, or expansion of the suspicious findings on serial imaging studies.

\section{References}

1. Yeo TC, Malouf JF, Oh JK, Seward JB. Clinical profile and outcome in 52 patients with cardiac pseudo-aneurysm. Ann Intern Med. 1998;128:299-305.

2. Milgalter E, Uretzky G, Levy P, Appelbaum A, Borman JB. Pseudo-aneurysm of the left ventricle. Thorac Cardiovasc Surg. 1987;35:20-25.

3. Vlodaver Z, Coe JI, Edwards JE. True and false left ventricular aneurysms. Propensity for the latter to rupture. Circulation. 1975;51:567-72.

\title{
Successful Fontan completion in a patient with noncompaction myocardium
}

\author{
Yoshinori Miyahara, $\mathrm{MD}, \mathrm{PhD}$, Shingo Kasahara, $\mathrm{MD}, \mathrm{PhD}$, Kozo Ishino, $\mathrm{MD}, \mathrm{PhD}$, \\ Shigeru Sakurai, MD, and Shunji Sano, MD, PhD, Okayama, Japan
}

Noncompaction myocardium is a rare cardiomyopathy that represents numerous prominent trabeculations and deep intertrabecular recesses mainly in the left ventricle $(\mathrm{LV}) .{ }^{1}$ The cause of noncompaction myocardium is thought to be abnormal cessation of endomyocardial morphogenesis. Although noncompaction myocardium has been commonly described in association with other structural heart abnormalities, ${ }^{2}$ few operative outcomes have been reported. We describe a successful surgical case of tricuspid atresia with noncompaction myocardium.

\section{CLINICAL SUMMARY}

A 22-year-old man was referred to the Okayama University Graduate School of Medicine, Dentistry, and Pharmaceutical Sciences for dyspnea on exertion and progressive cyanosis (oxygen saturation $\left[\mathrm{SPO}_{2}\right] 75 \%$ on room air, New York Heart Association II). Tricuspid atresia with normally related great vessels, pulmonary stenosis, and noncompaction myocar-

\footnotetext{
From the Department of Cardiovascular Surgery, Okayama University Graduate School of Medicine, Dentistry, and Pharmaceutical Sciences, Okayama, Japan. Disclosures: None.

Received for publication Sept 27, 2008; accepted for publication Feb 24, 2009; available ahead of print June 12, 2009.

Address for reprints: Yoshinori Miyahara, MD, PhD, Department of Cardiovascular Surgery, Okayama University Graduate School of Medicine, Dentistry, and Pharmaceutical Sciences, 2-5-1 Shikata-cho, Okayama, 700-8558, Japan (E-mail: giten@fd5.so-net.ne.jp).

J Thorac Cardiovasc Surg 2010;139:e85-7

0022-5223/\$36.00

Copyright (c) 2010 by The American Association for Thoracic Surgery doi:10.1016/j.jtcvs.2009.02.056
}

dium had been diagnosed in the patient at birth. On admission, the patient was active $(156 \mathrm{~cm}, 33 \mathrm{~kg})$ with a normal cardiothoracic ratio and sinus rhythm with incomplete left bundle branch block. Echocardiogram showed spongiform myocardium only in the LV myocardium, large atrial and ventricular communications, and an ejection fraction of $40 \%$. Angiogram demonstrated a single left coronary artery, markedly protuberant trabeculations, and deep intertrabecular recesses in the whole LV (Figure 1, $A, B$ ), as observed by computed tomography (Figure 1,C). Hemodynamic data showed a mean superior vena cava (SVC) and inferior vena cava (IVC) pressure of $8 \mathrm{~mm} \mathrm{Hg}$, a mean pulmonary artery (PA) pressure of $11 \mathrm{~mm} \mathrm{Hg}$, PA resistance index of 1.7 Wood unit $/ \mathrm{m}^{2}, \mathrm{LV}$ end-diastolic pressure of $7 \mathrm{~mm} \mathrm{Hg}$, and a pulmonary to systemic flow ratio of 0.96 . In view of the social activity of this young patient and preserved cardiac function, we decided to conduct a staged-Fontan surgery.

The patient was then scheduled to undergo an elective bidirectional Glenn shunt. Anastomosis between the SVC and the right PA was performed without cardiac arrest, using temporary bypass from the SVC through the right atrium, leaving pulmonary antegrade flow. Weaning from circulatory bypass was uneventful, as was the postoperative course, maintaining an $\mathrm{SPO}_{2}$ in the mid 80s until total cavopulmonary connection.

Two years after the bidirectional Glenn shunt, however, this patient had intracerebral hemorrhage caused by arteriovenous malformation and received an emergency coil embolization. He recovered without physical and mental disability. During admission, incidental Holter electrocardiogram 